\title{
How PEDOT:PSS solutions produce satellite-free inkjets
}

\author{
Stephen D. Hoath ${ }^{* a}$, Sungjune Jung ${ }^{a 1}$, Wen-Kai Hsiao ${ }^{a}$ and Ian M. \\ Hutchings ${ }^{a}$
}

${ }^{a}$ Department of Engineering, University of Cambridge, 17 Charles Babbage Road, Cambridge CB3 0FS, UK

* corresponding author Stephen Daniel Hoath

E: sdh35@cam.ac.uk T: +44 1223764626 F: +44 1223464217

Sungjune Jung

E: sjj37@cam.ac.uk T: +44 1223347491

Wen-Kai Hsiao

E: wkh26@cam.ac.uk T: +44 1223765600

Ian M. Hutchings

E: imh2@cam.ac.uk T: +44 1223765217

\begin{abstract}
Satellite droplets are unwanted in inkjet printing and various approaches have been suggested for their reduction. Low jetting speeds limit applications of the process. Added surfactants for wetting and conductivity enhancement may help but dynamic surface tension effects may counteract improvements. A higher fluid viscosity delays ligament break-up, but also leads to slower jets, while viscoelasticity reduces satellite formation only in certain cases. We show here that aqueous solutions of PEDOT:PSS (1:2.5 by weight) are strongly shear-thinning. They exhibit low viscosity within the printing nozzle over a wide range of jet speeds, yet rapidly $(<100 \mu \mathrm{s})$ recover a higher viscosity at the low shear rates applicable once the jet has formed, which give the benefit of delayed satellite formation. The delay over a $0.8 \mathrm{~mm}$ standoff distance can be sufficient to completely suppress satellites, which is significant for many printing applications.

Keywords:

PEDOT:PSS (poly(3,4 ethylenedioxythiophene:(polystyrene-sulfonate)); surfactant; DoD (drop-on-demand) inkjet printing; satellites; viscoelastic; shear-thinning fluid

\author{
Abbreviations \\ PEDOT:PSS poly(3,4ethylenedioxythiophene):(polystyrene-sulphonate) \\ DoD drop-on-demand \\ ${ }^{1}$ Present Address \\ Department of Physics, University of Cambridge, J.J. Thompson Avenue, Cambridge \\ CB3 0HE, UK
}




\section{Introduction}

There is increasing interest in the use of DoD (drop-on-demand) inkjet printing as an additive manufacturing process. Satellite drops, which often follow each fast-moving main drop ejected from the print-head nozzle, are undesirable because they are far more readily misdirected by aerodynamic and electrostatic forces and can thereby degrade the printing resolution and in the fabrication of conductive features can even cause bridging between neighbouring tracks, device failure and low production yield. Air, moisture and dust carried by satellite drops onto the substrate, film and/or main drop may also degrade the final film quality and device performance.

Aqueous dispersions of PEDOT:PSS with or without surfactants have been successfully deposited by inkjet printing for some years to form transparent conductors in printed electronics [1-8], but it has only recently become apparent that their main and satellite drop formation across a wide range of speeds is unusual in comparison with most other printable polymeric fluids.

The present quantitative study of satellite drop formation investigates reasons for excellent jetting of aqueous PEDOT:PSS solutions over a wide range of jet speeds and provides the first report of shear-thinning behaviour for PEDOT:PSS contributing to its excellent "jettability".

\section{Materials and methods}

Aqueous solutions at nominal values $1.0 \mathrm{wt} \%, 0.7 \mathrm{wt} \%$ and $0.5 \mathrm{wt} \%$ PEDOT:PSS were prepared from a commercial solution (Heraeus Clevios PH 1000, $1.1 \mathrm{wt} \%$ solids in water, 1:2.5 PEDOT:PSS by weight, quoted gel particle size $\mathrm{d}_{50}=25 \mathrm{~nm}$ ), with and without low molecular weight $(<1 \mathrm{kDa})$ surfactants $(0.23 \mathrm{wt} \%$ Dynol 607 and 0.27 wt $\%$ Zonyl FSO-100). Newtonian glycerol/water mixtures were prepared with glycerol concentrations of $39 \mathrm{wt} \%$ (G39W61, viscosity $3.4 \mathrm{mPa} \mathrm{s}$ at $\left.21^{\circ} \mathrm{C}\right)$ and 60 $\mathrm{wt} \%$ (G60W40, $10.3 \mathrm{mPa}$ s at $\left.21^{\circ} \mathrm{C}\right)$.

Measured surface tensions (at $21^{\circ} \mathrm{C}$ ) of all the PEDOT:PSS and Newtonian solutions were $21 \pm 2 \mathrm{mN} \mathrm{m}^{-1}$ and $70 \pm 3 \mathrm{mN} \mathrm{m}^{-1}$, with and without surfactants, respectively.
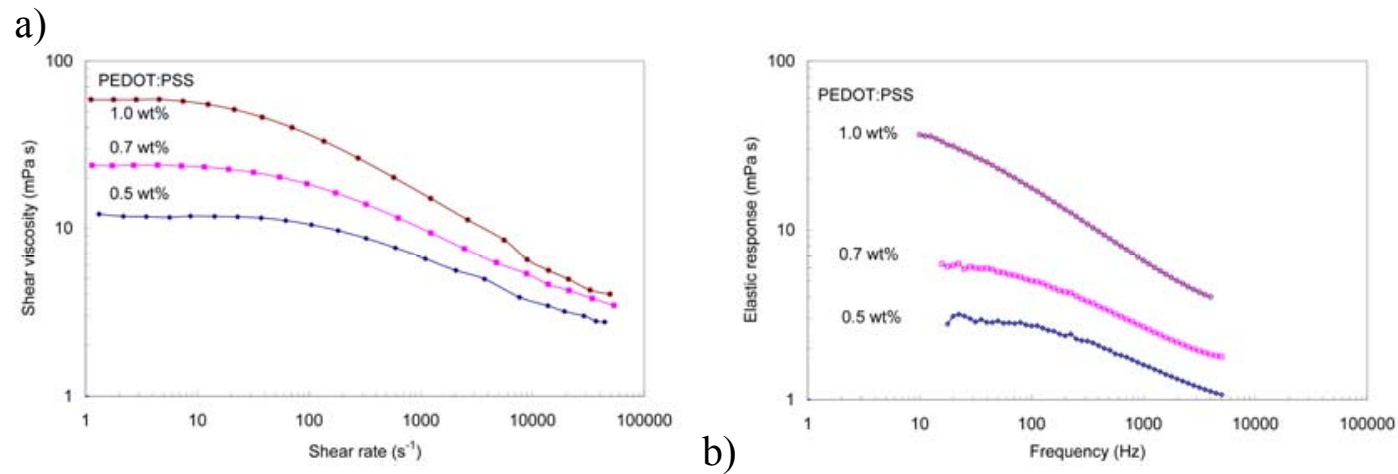

Figure 1: Measured values of (a) shear viscosity ( $\mathrm{mPa} \mathrm{s}$ ) vs. shear rate $\left(\mathrm{s}^{-1}\right)$ and (b) elastic response (mPas) vs. frequency $(\mathrm{Hz})$ for the PEDOT:PSS solutions indicated. 
Rheological measurements were performed with a parallel plate rotational rheometer at shear rates up to $40 \mathrm{kHz}$ [9] and with a piezo axial vibrator [10]. Results at $25^{\circ} \mathrm{C}$ are shown in Figure 1 (a) and (b), respectively. All PEDOT:PSS solutions significantly shear-thinned, but the presence of surfactants did not affect the rheological behaviour. For the most concentrated solution the viscosity fell from about $60 \mathrm{mPa}$ s at low shear rate to about $4 \mathrm{mPa} s$ at the highest measured shear rates. The PEDOT:PSS fluids also exhibited an elastic response steadily reducing with increase of frequency.

The fluids were jetted at $21^{\circ} \mathrm{C}$ in DoD mode from a single-nozzle piezoelectric printhead (type ABP with diamond-like carbon coated nozzle, MicroFab Technologies, Plano, TX, USA) with a nominal exit diameter of $40 \mu \mathrm{m}$. The print-head was driven by a uni-polar waveform ( $5 \mu$ s rise time, $15 \mu$ s dwell time, $5 \mu$ s fall time) with a range of drive voltages ( $20 \mathrm{~V}$ to $75 \mathrm{~V})$, which resulted in main drop speeds up to $15 \mathrm{~m} \mathrm{~s}^{-1}$.

Images of the jets were formed with a CCD camera, using an automated nano-flash illumination and data capture system [11]. The jet tip and tail speeds and the average satellite production were determined from images recorded at different time delays.

\section{Results}

Solutions jetted without surfactant produced satellites following early break up of the jet immediately behind the main head, but jetting with surfactant is of greater interest.

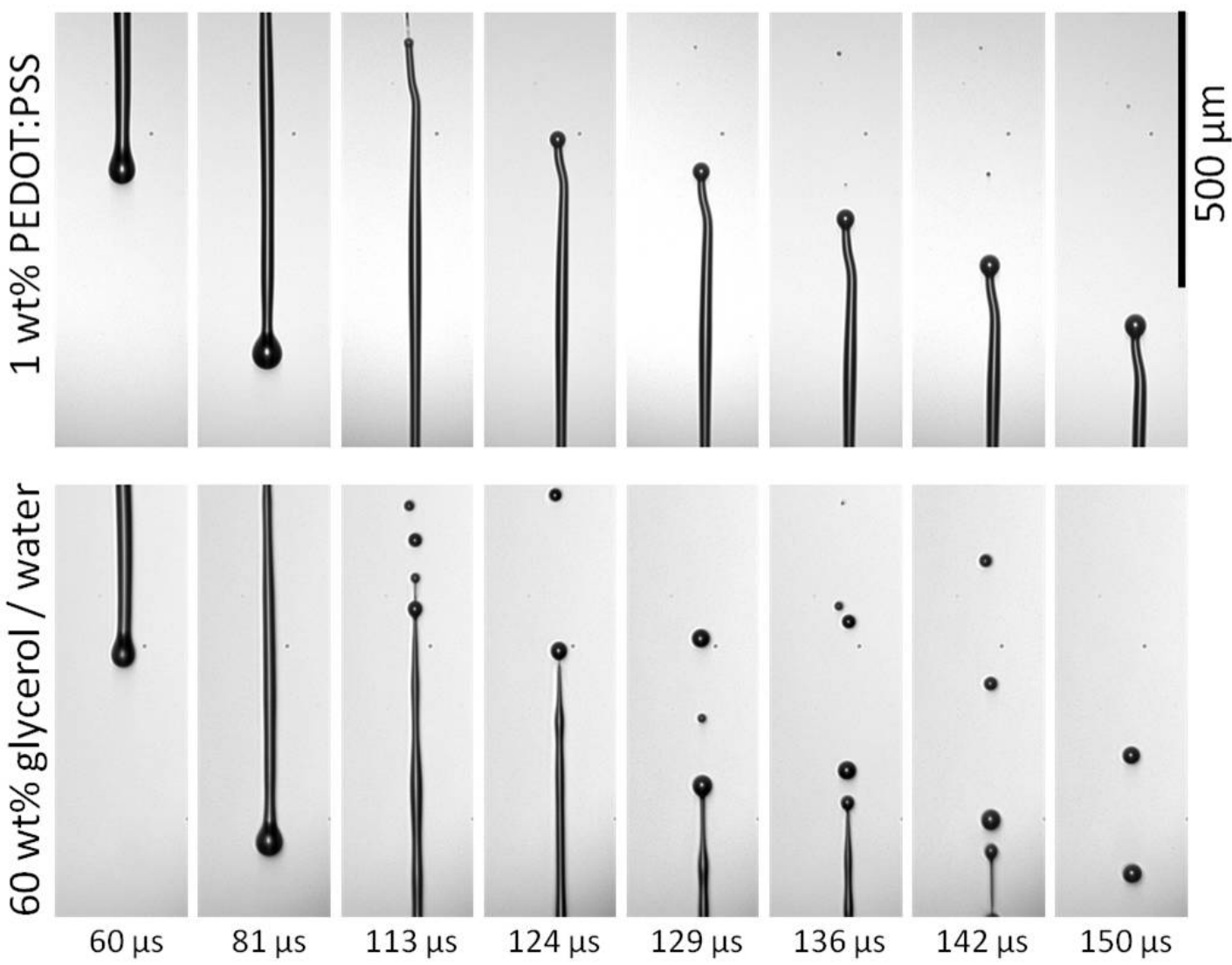

Figure 2. Jets formed at $65 \mathrm{~V}$ drive voltage by nominal $1 \mathrm{wt} \%$ PEDOT:PSS and G60W40 at the delay times shown. The nozzle exit was located just above the top edge of each image, and the jets were traveling vertically downwards. 


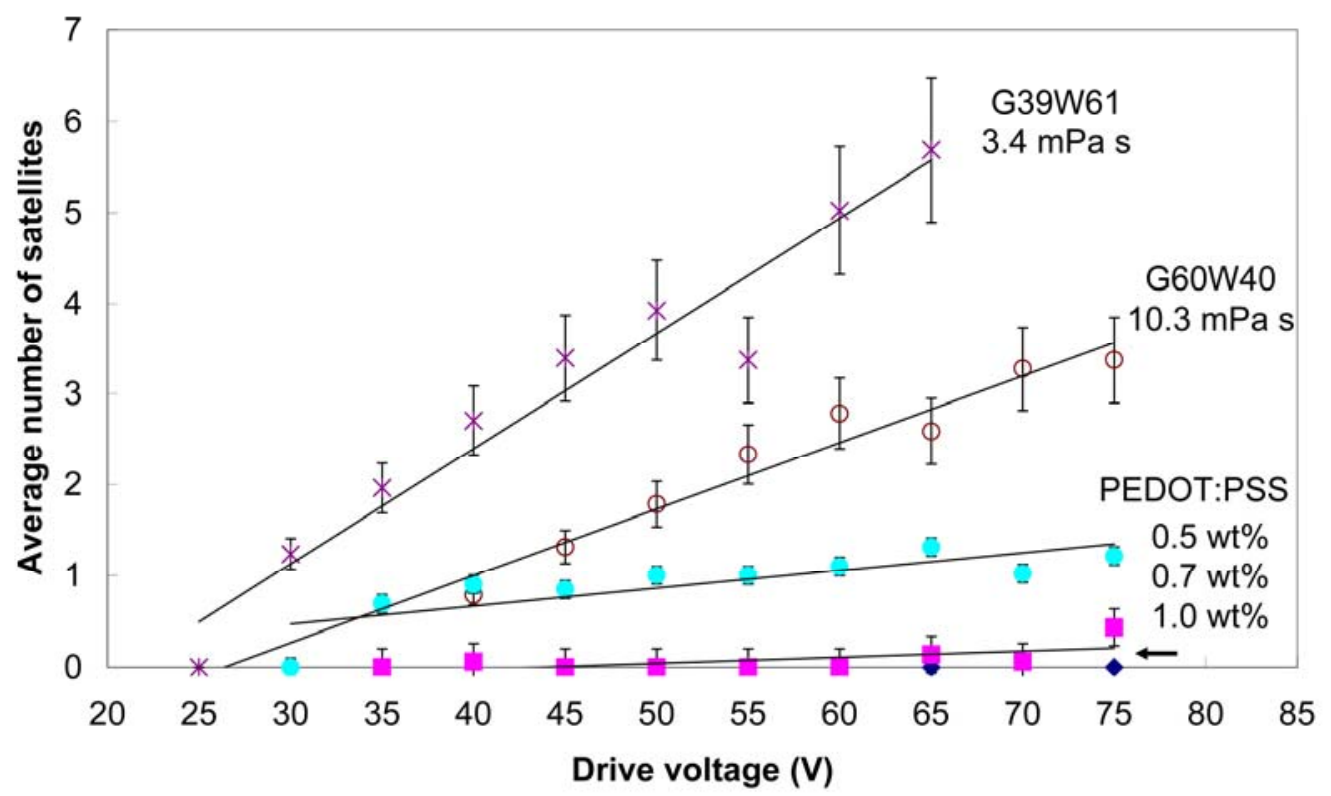

Figure 3. The average numbers of satellites, within $0.8 \mathrm{~mm}$ of the nozzle, from jetting of the PEDOT:PSS and Newtonian fluids with surfactants at a range of drive voltages. The lines are drawn to guide the eye. $1.0 \mathrm{wt} \%$ PEDOT:PSS had no visible satellites.

Figure 2 compares images of jets formed with the nominal $1 \mathrm{wt} \%$ PEDOT:PSS and G60W40 fluids with surfactant at $65 \mathrm{~V}$ drive voltage and drop speeds of $12 \pm 1 \mathrm{~m} \mathrm{~s}^{-1}$. These conditions were chosen to favour satellite drop in low viscosity fluids. Images at certain delay times demonstrate similar jet speeds (at $60,81 \mu \mathrm{s}$ ) and tail speeds (at the later times). Satellites are visible with the G60W40 fluid but none for the $1 \mathrm{wt} \%$ PEDOT:PSS fluid. The average numbers of satellites over a $0.8 \mathrm{~mm}$ stand-off distance of the DoD nozzle are plotted, for the solutions with surfactants, against nozzle drive voltage in Figure 3.

For all three concentrations of PEDOT:PSS, the production of satellites was delayed in comparison with the behaviour of the glycerol/water mixtures, at all main drop speeds up to about $15 \mathrm{~m} \mathrm{~s}^{-1}$, leading to much lower numbers of satellites in the field of view. For nominal $1 \mathrm{wt} \%$ PEDOT:PSS solution, no satellite drops were found at all.

\section{Discussion}

Figure 1a shows the steep reduction of the aqueous PEDOT:PSS viscosity with increasing shear rate. The low shear rate viscosity is such that the nominal $1 \mathrm{wt} \%$ fluid would not be jettable through an ink-jet nozzle. However this fluid does jet with a similar ease to the Newtonian $10 \mathrm{mPa}$ s $60 \mathrm{wt} \%$ glycerol in water, implying a very rapid change of the aqueous PEDOT:PSS structure over the duration of the nozzle transit time. The MicroFab nozzle converges to $40 \mu \mathrm{m}$ diameter within a $1 \mathrm{~mm}$ length and therefore at $6 \mathrm{~m} \mathrm{~s}^{-1}$ fluid speed the shear thinning inside the nozzle must occur within $<<160 \mu \mathrm{s}$. (Numerical simulations of shear-thinning fluid jetting behaviour gave times $<10 \mu \mathrm{s}$.) Jetting through other print head nozzles requires shear thinning on similar timescales. 
Figure $1 \mathrm{~b}$ contrasts with the marked elasticity increase with frequency for dilute linear polymer solutions studied in earlier work [12] that reduced the number of satellites in only certain cases. Figure 2 shows that under the conditions of this experiment the speeds of the main drops from the $1 \mathrm{wt} \%$ PEDOT:PSS solution and the Newtonian fluid were similar $\left(\approx 12 \mathrm{~m} \mathrm{~s}^{-1}\right)$. At the later times the tails of the PEDOT:PSS jet are not significantly advanced (downwards) beyond those of the Newtonian fluid that had already broken up into satellite drops. Figures $1 \mathrm{~b}$ and 2 imply that elastic responses of the PEDOT:PSS solutions can not significantly contribute to the satellite production. As surfactants take time (typically $>10^{-2} \mathrm{~s}$ for dynamic surface tension lowering [4]) to reach fresh surfaces, ink-jet satellite production should be enhanced for all of the aqueous solutions. However the major difference here between shear thinning (Figure 1a) and Newtonian fluids in terms of satellite formation could be due to a difference in their viscosity at the low shear rates relevant to fluid motion in the ligament.

Radial collapse of a Newtonian liquid ligament for radius $R$, viscosity $\eta$ and surface tension $\sigma$, takes time $\sim \eta \mathrm{R} /(0.0304 \sigma)$ [13]. Under rapid DoD jetting conditions (with dynamic surface tension $\sigma$ for these solutions between 21 and $72 \mathrm{mN} \mathrm{m}^{-1}$ ), the radial collapse time for $1 \mathrm{wt} \%$ PEDOT:PSS $(\eta=30 \mathrm{mPa} \mathrm{s})$ is about $150-300 \mu \mathrm{s}$ for $R=10 \mu \mathrm{m}$, comparable with the time taken for a $6 \mathrm{~m} \mathrm{~s}^{-1}$ drop to cross a $1 \mathrm{~mm}$ standoff distance on to the substrate, while the Newtonian fluid G60W40 ( $\eta=10 \mathrm{mPa}$ s) would form satellites within 25-50 $\mu$ s. Figures 1, 2 and 3 appear fully consistent with all these factors. Furthermore, irrespective of the dynamic surface tension value, the absence of necking for the jetted $1 \mathrm{wt} \%$ PEDOT:PSS in Figure 2 implies that the ligament viscosity returned to above $50 \%$ of the low shear-rate viscosity within about $100 \mu \mathrm{s}$ after the jet break-off time. Thus the recovery timescale for the aqueous $1 \mathrm{wt} \%$ PEDOT:PSS fluid is very rapid, typically $<100 \mu \mathrm{s}$, and well beyond the $>1 \mathrm{~s}$ capabilities of conventional rheometry.

While the present discussion was limited to a given commercial formulation (1:2.5) of a particular fluid (aqueous PEDOT:PSS with size distribution parameter $\mathrm{d}_{50}=25 \mathrm{~nm}$ ), for which other information is given in [5], it is clearly of great interest to establish a more robust characterization of aqueous PEDOT:PSS, and other shear thinning fluids, as a means of improving the general quality of ink-jetting for many applications. We intend to address this expansion as part of our continuing research program.

\section{Conclusions}

Quantitative evidence and explanations for the suppression of satellites from rapidly jetted fluids has been provided for the first time. Shear thinning is responsible for this and such fluids will assist other jetting applications. The remarkable successes in jetting PEDOT:PSS solutions for electronics applications are now better understood. Further work on the robust characterisation of different formulations would be useful.

\section{Acknowledgements}

This work was supported by the UK EPSRC through grant number EP/H018913/1 (Innovation in Industrial Ink-jet Technology). SJ acknowledges materials from the Cavendish Laboratory. We thank Oliver Harlen, Neil Morrison, Simon Butler, Clare Conboy and Adrian Hill for suggestions and help with the fluid characterisation. Heraeus Conductive Polymer Division quoted the $\mathrm{d}_{50}$ value for Clevios PH 1000. 


\section{References}

[1] C.W. Sele, T. von Werne, R.H. Friend, H. Sirringhaus, Lithography-free, selfaligned inkjet printing with sub-hundred-nanometer resolution, Advanced Materials 17 (2005) 997-1001

[2] S.H. Eom, S. Senthilarasu, P. Uthirakumar, S.C. Yoon, J. Lim, C. Lee, H.S. Lim, J. Lee, S.-H. Lee, Polymer solar cells based on inkjet-printed PEDOT:PSS layer, Organic Electronics 10 (2009) 536-542

[3] M.-W. Lee, M.-Y. Lee, J.C. Choi, J.-S. Park, C.-K. Song, Fine patterning of glycerol-doped PEDOT:PSS on hydrophobic PVP dielectric with ink jet for source and drain electrode of OTFTs, Organic Electronics 11 (2010) 854-859

[4] M. Ren, H. Gorter, J.Michels, R. Andriessen, Ink Jet Technology for Large Area Organic Light-Emitting Diode and Organic Photovoltaic Applications, J. Imaging Sci. Technol. 55 (2011) 040301-040301-6

[5] A. Elschner, W. Loevenich, A. Eiling, J. Bayley, ITO Alternative: solution deposited Clevios ${ }^{\mathrm{TM}}$ PEDOT:PSS for transparent conductive applications, Heraeus Precious Metals GmbH \& Co KG, Conductive Polymers Division, Leverkusen, Germany, Trade Article June $6^{\text {th }} 2012$

[6] P. Wilson, C. Lekakou, J.F. Watts, A comparative assessment of surface microstructure and electrical conductivity dependence on co-solvent addition in spin coated and inkjet printed poly(3,4-ethylenedioxythiophene):polystyrene sulphonate (PEDOT:PSS), Organic Electronics 13 (2012) 409-418

[7] Z. Xiong, C. Liu, Optimization of inkjet printed PEDOT:PSS thin films through annealing processes, Organic Electronics 13 (2012) 1532-1540

[8] Y. Xia, J. Ouyang, Significant different conductivities of the two grades of Poly(3,4-ethylenedioxythiophene):Poly(styrenesulphonate), Clevios P and Clevios PH1000, arising from different molecular weights, Appl. Mater. Interfaces 4 (2012) 4131-4140

[9] M.M. Cross, A. Kaye, Simple procedures for obtaining viscosity/shear rata data from a parallel disc viscometer, Polymer 28 (1987) 435-440

[10] D.C. Vadillo, T. R. Tuladhar, A. Mulji, M.R. Mackley, The rheological characterisation of linear viscoelasticity for ink jet fluids using Piezo Axial Vibrator (PAV) and Torsion Resonator (TR) rheometers, J. Rheol. 54 (2010) 781-795

[11] S. Jung, I.M. Hutchings, The impact and spreading of a small liquid drop on a non-porous substrate over an extended timescale, Soft Matter 8 (2012) 2686-2696;

[12] S.D. Hoath, G.D. Martin, J.R. Castrejón-Pita, I.M. Hutchings, Satellite formation in drop-on-demand printing of polymer solutions, Society for Imaging Science and Technology, Springfield VA, Technical proceedings of Non-Impact Printing conference 23 (2007) 331-335 
[13] S.D. Hoath, G.D. Martin, I.M. Hutchings, Effects of fluid viscosity on drop-ondemand inkjet break-off, Society for Imaging Science and Technology, Springfield VA, Technical proceedings of Non-Impact Printing conference 26 (2010) 10-13 